\title{
Prevalence of Workplace Physical Violence against Health Care Professionals by Patients and Visitors: A Systematic Review and Meta-Analysis
}

\author{
Yi-Lu Li, Rui-Qi Li, Dan Qiu and Shui-Yuan Xiao * \\ Department of Social Medicine and Health Management, Xiangya School of Public Health, \\ Central South University, Changsha 410078, Hunan, China; 176911065@csu.edu.cn (Y.-L.L.); \\ 176911062@csu.edu.cn (R.-Q.L.); 166911058@csu.edu.cn (D.Q.) \\ * Correspondence: xiaosy@csu.edu.cn; Tel.: +86-0731-84-805-459
}

Received: 10 December 2019; Accepted: 30 December 2019; Published: 1 January 2020

\begin{abstract}
Workplace physical violence against health care professionals perpetrated by patients and visitors has been a persistent problem worldwide. Prevalence estimates varied vastly across studies and there was a lack of quantitative syntheses of prevalence studies. This review aimed to quantify pooled one-year prevalence estimates at the global and regional levels. A systematic literature search was performed in the databases of PubMed, PsycINFO, Web of Science, and Embase between 1 January 2000 and 8 October 2018. Studies providing information about one-year prevalence of self-reported workplace physical violence against health care professionals perpetrated by patients or visitors were included. Heterogeneity between studies was evaluated using Cochran's chi-squared test (Cochran's Q) and $\mathrm{I}^{2}$ values. Subgroup analysis and meta-regression were used to explore heterogeneity. A total of 65 eligible studies reported one-year prevalence estimates for 61,800 health care professionals from 30 countries. The pooled one-year prevalence of workplace physical violence against health care professionals perpetrated by patients or visitors was $19.33 \%$ (95\% confidence interval (CI): $16.49-22.53 \%$ ) and the overall heterogeneity was high across studies. We noted geographic and staff categories variations for prevalence estimates through subgroup analysis. The meta-regression showed that sample size, type of health care setting, and quality score were significant moderators for heterogeneity. One in five health care professionals experienced workplace physical violence perpetrated by patients or visitors worldwide annually. Practical intervention was needed to ensure safety of health care professionals.
\end{abstract}

Keywords: health care professionals; workplace violence; physical violence; meta-analysis

\section{Background}

Workplace physical violence against health care professionals has been a persistent problem of health care environment worldwide [1,2]. Health care professionals include physicians, nurses, technicians, and other medical staff who are in direct contact with patients and visitors. In 2009, 10\% of workplace assaults victims were health care professionals in the United States. [3]. The World Health Organization (WHO) defined workplace violence as the incidents where staff were abused, threatened, or assaulted in the circumstances related to their work [4]. Workplace violence had an explicit or implicit impact on employees' safety, well-being, and health. Workplace violence can have multiple negative consequences that not only result in physical consequences [5], but also psychological consequences for health care professionals [6,7]. Additionally, workplace violence was associated with the intention to quit job [8], burnout [7], and decreased job satisfaction [9] among health care professionals. Those consequences of workplace violence can lead to decreased productivity and 
even affect the quality of care. Moreover, staff absence [10] and investment of defensive tactics (e.g., security guard and metal detector) [11] caused by workplace violence may virtually increase health costs. Therefore, workplace violence in health sectors affected not only the health care professionals themselves, but also the entire health care environment.

The WHO had classified workplace violence into physical violence and psychological violence. Physical violence was defined as physical force (e.g., beating, kicking, slapping, stabbing, shooting, pushing, biting, and pinching) against a person or groups that results in physical, sexual, or psychological harm [4]. Physical violence was the most serious type of violence against health care professionals in their workplace [12]. Health care professionals accounted for $1.2 \%$ of workplace homicide victims of the United States [3], and about $4.9-65 \%$ of health care professionals were physically injured in their workplace during an incident of workplace physical violence [5]. Work stress, patient expectations, and deteriorative patient-staff relationships were associated with workplace physical violence against health care professionals [13]. Before developing policies and interventions, it is important to understand the prevalence and severity of workplace physical violence against health care professionals.

In order to obtain relatively reliable pooled prevalence estimates, the research included in meta-analysis should be relatively consistent in definition. The definition of workplace physical violence was more consistent across studies $[7,14,15]$. Extensive studies have been conducted to explore the prevalence and severity of workplace physical violence against health care professionals perpetrated by patients and visitors. Estimates of one-year prevalence of workplace physical violence against health care professionals perpetrated by patients or visitors in general hospital ranged from $2.75 \%$ in Thailand [16] to $74.42 \%$ in the United States [17]. Only a few of the systematic reviews have synthesized the results of prevalence studies. Those systematic reviews mainly focused on high-risk health care sectors [18-20], specific professional group [21], or specific country [22]. There was still a need for a systematic review that included all health care sectors, diverse health care professional types, and multiple countries. In addition, workplace physical violence in health sectors was mainly perpetrated by patients and visitors $[1,23]$. However, co-workers or superiors may also be the perpetrators of workplace physical violence against health care professionals. The nature of workplace physical violence perpetrated by co-workers or superiors was distinctly different from that perpetrated by patients or visitors. However, numerous studies did not report who perpetrated the workplace violence. Most of the systematic reviews did not describe the identity of the perpetrators [18-22]. Therefore, the prevalence of workplace physical violence against health care professionals by patients and visitors is still not clear and there is a lack of quantitative synthesized results. Considering the limitations of previous research, our study aimed to synthesize the results of workplace physical violence against health care professionals by patients and visitors.

To address the need for global estimates of prevalence of workplace physical violence against health care professionals perpetrated by patients or visitors, we did a meta-analysis of relevant studies around the world. We also aimed to understand how the methodological characteristics (i.e., sample size, response rate, method of data collection, sampling method) and contextual factors (i.e., region, health care setting) influenced the variations in prevalence estimates. A systematic literature search was performed. Possible relevant studies were screened based on strict eligibility criteria. Quality of eligible studies was assessed. Quantitative synthesized one-year prevalence of workplace physical violence against health care professionals perpetrated by patients and visitors was obtained by the meta-analysis.

\section{Methods}

\subsection{Search Strategy and Selection Criteria}

This meta-analysis was performed according to the Preferred Reporting Items for Systematic Reviews and Meta-Analyses (PRISMA) guidelines (see Table S1, Supplementary Materials). The following four academic databases were searched between 1 January 2000 and 8 October 2018: PubMed, 
PsycINFO, Web of Science, and Embase. The search strategy was developed and adjusted for each database with a combination of free text and controlled vocabulary terms. The following search terms were used: "physical violence" (including "physical violence", "workplace violence", and "occupational violence")", "health care professional" (including "health care professional" ", "nurse "doctor*", "physician*", and "health care worker*"), and "prevalence" (including "prevalence", "incidence", "cross-sectional", and "cohort"). A full list of the search terms is provided in Table S2, Supplementary Materials. Additionally, reference lists of eligible studies were manually screened for any relevant studies.

Studies were independently screened by two reviewers (Y.-L.L. and R.-Q.L.) using the eligibility criteria described below. Studies were included if they meet the following criteria: (i) provided one-year prevalence of self-reported workplace physical violence against health care professionals perpetrated by patients or visitors; or (ii) reported definition and measurement of workplace physical violence. We excluded studies if they met the following criteria: (i) included medical student, cleaning staff, clerk, security, or administrative staff as participants; (ii) did not report perpetrators of the workplace physical violence; (iii) reported response rate $<20 \%$, or no response rate was reported; or (iv) was conference abstract, report, review, meta-analysis, letters, pilot study, protocol, or qualitative study. Workplace physical violence against health care professionals included beating, kicking, slapping, stabbing, shooting, pushing, biting, and pinching against health care professionals in their workplace [4]. We included the studies only based on self-reported rather than record or monitoring data. To avoid bias in data synthesis, we included studies with the same prevalence period (one-year) for the meta-analysis. When findings from iterations of the same survey were reported, we included the publication that provided the most data.

\subsection{Data Extraction and Quality Assessment}

Two researchers (Y.-L.L. and R.-Q.L.) independently extracted relevant data from eligible studies and a third researcher (D.Q.) cross checked for accuracy. The following data were extracted: author, year of publication, country of study, sample size, categories of health care professionals, sampling method, method of data collection, response rate, type of health care setting, region of health care setting, and one-year prevalence estimates of workplace physical violence perpetrated by patients or visitors.

The methodological quality was assessed using the eight-item Loney criteria (see Table S3, Supplementary Materials). Studies satisfying one item will be given one point and an overall score was calculated. Therefore, the overall score ranged from zero to eight points, with higher scores indicating a higher degree of quality.

\subsection{Data Analysis}

Statistical analyses were performed using the "meta" and "metafor" package of R version 3.5.2 (R Core Team, Vienna, Austria). Firstly, a normality test for the original study rates was performed to decide whether to transform the original rates. According to the normality testing results, a logit transformation method was used in this meta-analysis. Heterogeneity between studies was evaluated using Cochran's chi-squared test (Cochran's $\mathrm{Q}$ ) and $\mathrm{I}^{2}$ values. The significant heterogeneity between studies was assumed when $p<0.1$ or $\mathrm{I}^{2}>50 \%$ [24]. A random effects model was adopted to calculate the pooled one-year prevalence of workplace physical violence against health care professionals perpetrated by patients or visitors if significant heterogeneity was observed across studies; otherwise, a fixed-effects model was adopted. To investigate the possible sources of heterogeneity and variations of prevalence estimates, subgroup analyses were conducted based on following categories: WHO regions (Western Pacific vs. European vs. eastern Mediterranean vs. Americas vs. African vs. South-East Asia); income classification of each country based on the World Bank classification (high-income vs. upper-middle-income vs. lower-middle-income vs. low-income countries); year of publication (2000 2010 vs. 2011 2018); sample size ( $\leq 500$ vs. $>500$ ); response rate ( $\leq 50 \%$ vs. $>50 \%$ ); professional 
group (nurses vs. physicians); method of data collection (self-administered vs. face-to-face interview vs. telephone interview); gender (male vs. female); sampling method (all vs. random vs. convenience); type of health care setting (tertiary hospital vs. secondary hospital vs. primary care facilities vs. nursing home); region of health care setting (urban vs. rural/township vs. mixed); and quality score $(\leq 5$ vs. $>5$ ). Differences within each subgroup were compared using Cochran's chi-squared test (Cochran's Q).

To further explore the relevant factors influencing prevalence estimates, univariate meta-regression analysis was conducted including the following covariates: year of publication, income classification, sample size, response rate, method of data collection, professional group, region of health care setting, type of health care setting, and quality score. The multivariate meta-regression analysis included only significant variables $(p<0.05)$ in the regression model based on the result of the univariate analysis.

Publication bias was assessed by the Begg's rank test, and a Begg's funnel plot for a symmetry was presented. Sensitivity analysis was conducted by removing each study sequentially to assess the consistency of the prevalence estimates. All statistical analyses were two-tailed with a significance level of 0.05 .

\section{Results}

\subsection{Study Selection}

The database search initially generated 17,923 articles and 6678 duplicates were removed. After title and abstract screening, 10,699 irrelevant articles were excluded. A total of 546 potentially relevant full-text articles were independently assessed based on the selection criteria. Further, 481 studies were excluded because of the following reasons: duplicate articles or results $(n=7)$; reviews and conference abstracts $(n=136)$; used qualitative method only $(n=14)$; did not report definition or measurement $(n=26)$; had a response rate $<20 \%$ or did not report response rate $(n=16)$; did not provide workplace physical violence prevalence data $(n=84)$; did not reported perpetrators $(n=89)$; including medical student, cleaning staff, clerk, security, and administrative staff $(n=38)$; and no reported and/or not one-year prevalence period $(n=71)$. Finally, 65 eligible studies were included for the meta-analysis (Figure 1).

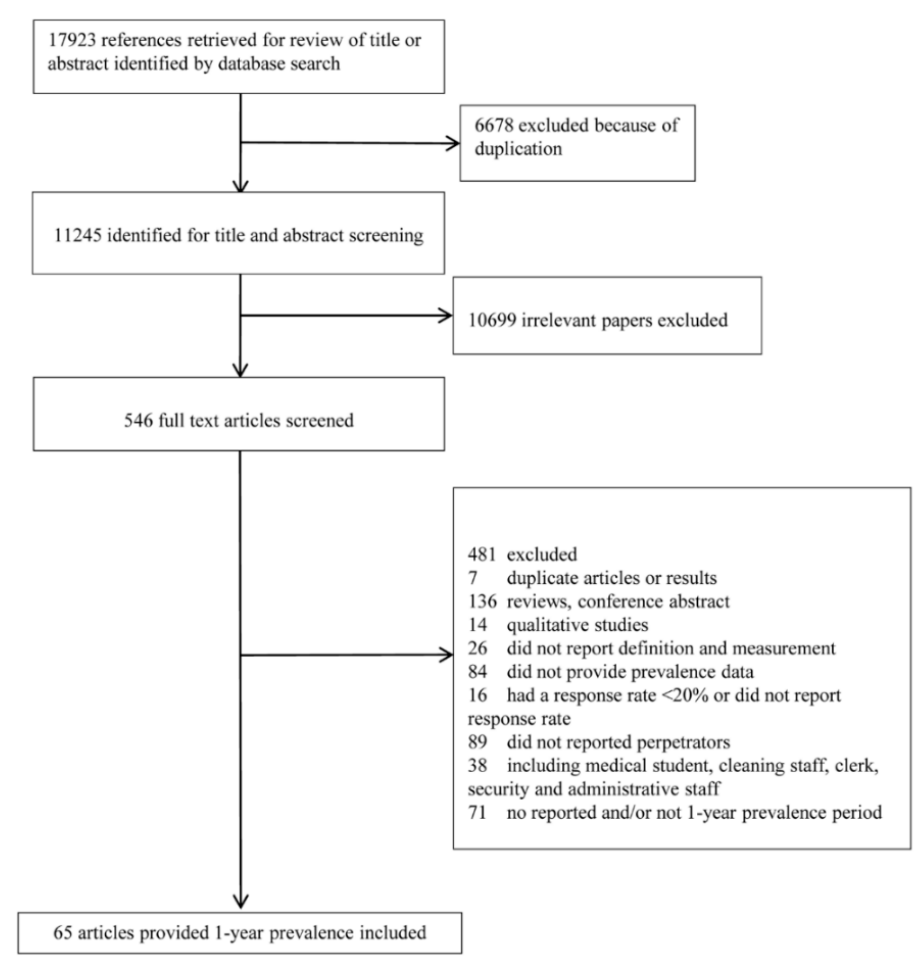

Figure 1. Preferred Reporting Items for Systematic Reviews and Meta-Analyses (PRISMA) flow chart of study identification process. 


\subsection{Study Characteristics}

A total of 61,800 health care professionals were included in this meta-analysis and the sample size ranged from 55 to 9218 participants per study. The eligible studies from 30 countries were geographically diverse, with 18 studies from the WHO region of Europe, 17 from the eastern Mediterranean, 14 from the western Pacific, 10 from the Americas, 4 from Africa, and 2 from Southeast Asia. Those countries were also divided into different income classification as follows: 32 studies from high-income countries, 20 from upper-middle-income countries, 11 from lower-middle-income countries, and 2 from low-income countries. Among eligible studies, 34 studies exclusively focused on nurses, 10 exclusively focused on physicians, and 16 focused on mixed staff categories. Quality scores ranged from three to eight points across studies. Minimum quality score of three was achieved in two studies and maximum quality score of eight was achieved in six studies. Seventeen studies were scored four points, 12 were scored five points, 18 were scored six points, and 10 were scored seven points (see Table 1). 
Table 1. Characteristics of prevalence studies included in meta-analysis. WHO, World Health Organization.

\begin{tabular}{|c|c|c|c|c|c|c|c|c|c|c|c|c|}
\hline Study & Country & WHO Region & Sample Size & Event & $\begin{array}{c}\text { Income } \\
\text { Classification }\end{array}$ & $\begin{array}{l}\text { Professional } \\
\text { Group }\end{array}$ & Setting & $\begin{array}{l}\text { Region of Health } \\
\text { Care Setting }\end{array}$ & $\begin{array}{l}\text { Method of Data } \\
\text { Collection }\end{array}$ & $\begin{array}{c}\text { Response } \\
\text { Rate }\end{array}$ & Sampling & $\begin{array}{l}\text { Quality } \\
\text { Score }\end{array}$ \\
\hline Eickson et al., 2000 [24] & U.S. & Americas & 55 & 31 & High income & nurses & $\begin{array}{l}\text { emergency } \\
\text { department }\end{array}$ & urban & self-administered & $98.00 \%$ & convenience & 4 \\
\hline May et al., 2002 [17] & U.S. & Americas & 86 & 64 & High income & nurses & general hospital & urban & self-administered & $68.80 \%$ & convenience & 3 \\
\hline Tolhurst et al., 2003 [25] & Australia & $\begin{array}{l}\text { Western } \\
\text { Pacific }\end{array}$ & 314 & 10 & High income & physicians & primary care & rural & self-administered & $51.80 \%$ & purposive & 4 \\
\hline $\begin{array}{c}\text { Kowalenko et al., } 2003 \\
\text { [26] }\end{array}$ & U.S. & Americas & 171 & 48 & High income & physicians & $\begin{array}{l}\text { emergency } \\
\text { department }\end{array}$ & mixed & self-administered & $68.40 \%$ & random & 4 \\
\hline $\begin{array}{c}\text { Gerberich et al., } 2004 \\
\text { [27] }\end{array}$ & U.S. & Americas & 3999 & 462 & High income & nurses & $\begin{array}{c}\text { Hospital/nursing } \\
\text { home/other setting }\end{array}$ & mixed & self-administered & $78.00 \%$ & random & 6 \\
\hline $\begin{array}{l}\text { Winstanley et al., } 2004 \\
\text { [28] }\end{array}$ & U.K. & European & 375 & 104 & High income & nurses/physicians & general hospital & mixed & self-administered & $33.00 \%$ & all & 6 \\
\hline $\begin{array}{l}\text { Carmi-Iluz et al., } 2005 \\
\text { [29] }\end{array}$ & Israel & European & 177 & 16 & High income & physicians & hospital/community & mixed & self-administered & $88.50 \%$ & convenience & 4 \\
\hline Nijman et al., 2005 [30] & U.K. & European & 154 & 136 & High income & nurses & psychiatric & urban & self-administered & $39.00 \%$ & all & 4 \\
\hline $\begin{array}{l}\text { AbuAlRub et al., } 2007 \\
\text { [31] }\end{array}$ & Iraq & $\begin{array}{c}\text { Eastern } \\
\text { Mediterranean }\end{array}$ & 116 & 40 & $\begin{array}{l}\text { Upper middle } \\
\text { income }\end{array}$ & nurses & general hospital & urban & $\begin{array}{l}\text { face-to-face } \\
\text { interview }\end{array}$ & $100.00 \%$ & purposive & 5 \\
\hline $\begin{array}{l}\text { Lundstrom et al., } 2007 \\
{[\text { [32] }}\end{array}$ & Sweden & European & 120 & 30 & High income & $\begin{array}{l}\text { nurses/assistant } \\
\text { nurses/nurse's } \\
\text { aides }\end{array}$ & nursing home & urban & self-administered & $81.00 \%$ & I & 4 \\
\hline $\begin{array}{c}\text { Kamchuchat et al., } 2008 \\
\text { [16] }\end{array}$ & Thailand & $\begin{array}{l}\text { South-East } \\
\text { Asia }\end{array}$ & 545 & 15 & $\begin{array}{l}\text { Upper middle } \\
\text { income }\end{array}$ & nurses & general hospital & 1 & self-administered & $91.70 \%$ & all & 7 \\
\hline Gale et al., 2009 [33] & New Zealand & $\begin{array}{l}\text { Western } \\
\text { Pacific }\end{array}$ & 197 & 37 & High income & physicians & psychiatric & mixed & self-administered & $63.90 \%$ & all & 4 \\
\hline Stubbs et al., 2009 [34] & U.K. & European & 116 & 28 & High income & physicians & psychiatric & mixed & self-administered & $65.00 \%$ & all & 4 \\
\hline $\begin{array}{c}\text { Galinsky et al., } 2010 \\
\text { [35] }\end{array}$ & U.S. & Americas & 677 & 31 & High income & $\begin{array}{l}\text { nurses/assistant } \\
\text { nurses/nurse's } \\
\text { aides }\end{array}$ & home healthcare & urban & $\begin{array}{l}\text { face-to-face } \\
\text { interview }\end{array}$ & $64.00 \%$ & convenience & 4 \\
\hline Hahn et al., 2010 [36] & Switzerland & European & 291 & 122 & High income & nurses & general hospital & I & self-administered & $71.00 \%$ & purposive & 4 \\
\hline Tak et al., 2010 [37] & U.S. & Americas & 2888 & 982 & High income & assistant nurses & nursing home & l & $\begin{array}{l}\text { telephone } \\
\text { interview }\end{array}$ & $70.60 \%$ & random & 7 \\
\hline $\begin{array}{l}\text { Zampieron et al., } 2010 \\
\text { [38] }\end{array}$ & Italy & European & 595 & 45 & High income & nurses & general hospital & urban & self-administered & $85.00 \%$ & convenience & 6 \\
\hline $\begin{array}{c}\text { Abualrub et al., } 2011 \\
\text { [39] }\end{array}$ & Jordan & $\begin{array}{c}\text { Eastern } \\
\text { Mediterranean }\end{array}$ & 422 & 85 & $\begin{array}{l}\text { Upper middle } \\
\text { income }\end{array}$ & nurses & general hospital & I & self-administered & $84.40 \%$ & convenience & 5 \\
\hline Behnam et al., 2011 [40] & U.S. & Americas & 263 & 48 & High income & physicians & $\begin{array}{l}\text { emergency } \\
\text { department }\end{array}$ & mixed & self-administered & $97.00 \%$ & random & 6 \\
\hline $\begin{array}{c}\text { Campbell et al., } 2011 \\
\text { [41] }\end{array}$ & U.S. & Americas & 2166 & 379 & High income & nurses & hospital/elder care & urban & self-administered & $52.00 \%$ & all & 5 \\
\hline $\begin{array}{l}\text { Esmaeilpour et al., } 2011 \\
\text { [42] }\end{array}$ & Iran & $\begin{array}{c}\text { Eastern } \\
\text { Mediterranean }\end{array}$ & 186 & 35 & $\begin{array}{l}\text { Upper middle } \\
\text { income }\end{array}$ & nurses & $\begin{array}{l}\text { emergency } \\
\text { department }\end{array}$ & urban & self-administered & $94.80 \%$ & I & 6 \\
\hline Pai et al., 2011 [43] & China Taiwan & $\begin{array}{l}\text { Western } \\
\text { Pacific }\end{array}$ & 545 & 89 & High income & nurses & health care setting & mixed & self-administered & $77.90 \%$ & random & 5 \\
\hline Petzall et al., 2011 [44] & Switzerland & European & 132 & 21 & High income & $\begin{array}{l}\text { ambulance } \\
\text { personnel }\end{array}$ & ambulance stations & mixed & self-administered & $79.00 \%$ & convenience & 8 \\
\hline Pinar et al., 2011 [45] & Turkey & European & 255 & 191 & $\begin{array}{l}\text { Upper middle } \\
\text { income }\end{array}$ & nurses & $\begin{array}{l}\text { emergency } \\
\text { department }\end{array}$ & urban & self-administered & $96.22 \%$ & all & 4 \\
\hline Ukpong et al., 2011 [46] & Nigeria & African & 101 & 34 & $\begin{array}{l}\text { Lower middle } \\
\text { income }\end{array}$ & nurses/physicians & psychiatric & urban & self-administered & $84.20 \%$ & 1 & 5 \\
\hline $\begin{array}{c}\text { Khoshknab et al., } 2012 \\
\text { [47] }\end{array}$ & Iran & $\begin{array}{c}\text { Eastern } \\
\text { Mediterranean }\end{array}$ & 183 & 124 & $\begin{array}{l}\text { Upper middle } \\
\text { income }\end{array}$ & nurses & psychiatric & urban & self-administered & $91.50 \%$ & random & 4 \\
\hline
\end{tabular}


Table 1. Cont.

\begin{tabular}{|c|c|c|c|c|c|c|c|c|c|c|c|c|}
\hline Study & Country & WHO Region & Sample Size & Event & $\begin{array}{c}\text { Income } \\
\text { Classification }\end{array}$ & $\begin{array}{l}\text { Professional } \\
\text { Group }\end{array}$ & Setting & $\begin{array}{l}\text { Region of Health } \\
\text { Care Setting }\end{array}$ & $\begin{array}{c}\text { Method of Data } \\
\text { Collection }\end{array}$ & $\begin{array}{c}\text { Response } \\
\text { Rate }\end{array}$ & Sampling & $\begin{array}{l}\text { Quality } \\
\text { Score }\end{array}$ \\
\hline $\begin{array}{l}\text { Magnavita et al., } 2011 \\
\text { [48] }\end{array}$ & Italy & European & 275 & 34 & High income & nurses & general hospital & l & self-administered & $94.20 \%$ & l & 6 \\
\hline Hahn et al., 2012 [49] & Switzerland & European & 2495 & 422 & High income & $\begin{array}{l}\text { health care } \\
\text { professionals }\end{array}$ & general hospital & l & self-administered & $51.50 \%$ & all & 4 \\
\hline Joa et al., 2012 [50] & Norway & European & 527 & 67 & High income & $\begin{array}{l}\text { health care } \\
\text { professionals }\end{array}$ & primary care & mixed & $\begin{array}{l}\text { telephone } \\
\text { interview }\end{array}$ & $75.00 \%$ & all & 6 \\
\hline Kitaneh et al., 2012 [51] & Palestine & $\begin{array}{c}\text { Eastern } \\
\text { Mediterranean }\end{array}$ & 240 & 43 & $\begin{array}{l}\text { Lower middle } \\
\text { income }\end{array}$ & nurses/physicians & general hospital & 1 & self-administered & $88.70 \%$ & random & 6 \\
\hline Gascon et al., 2013 [52] & Spain & European & 1826 & 293 & High income & $\begin{array}{l}\text { health care } \\
\text { professionals }\end{array}$ & $\begin{array}{l}\text { hospital/primary } \\
\text { care center }\end{array}$ & mixed & self-administered & $76.00 \%$ & random & 6 \\
\hline Hills et al., 2013 [53] & Australia & $\begin{array}{l}\text { Western } \\
\text { Pacific }\end{array}$ & 9218 & 2548 & High income & physicians & health care setting & mixed & self-administered & $60.90 \%$ & all & 7 \\
\hline $\begin{array}{l}\text { A.LBashtawyM et al., } \\
2013 \text { [54] }\end{array}$ & Jordan & $\begin{array}{c}\text { Eastern } \\
\text { Mediterranean }\end{array}$ & 227 & 24 & $\begin{array}{l}\text { Lower middle } \\
\text { income }\end{array}$ & nurses & $\begin{array}{l}\text { emergency } \\
\text { department }\end{array}$ & 1 & self-administered & $72.50 \%$ & convenience & 6 \\
\hline Zafar et al., 2013 [55] & Pakistan & $\begin{array}{l}\text { Eastern } \\
\text { Mediterranean }\end{array}$ & 266 & 37 & $\begin{array}{l}\text { Lower middle } \\
\text { income }\end{array}$ & nurses/physicians & $\begin{array}{l}\text { emergency } \\
\text { department }\end{array}$ & urban & self-administered & $86.00 \%$ & all & 4 \\
\hline $\begin{array}{c}\text { AbuAlRub et al., } 2014 \\
\text { [56] }\end{array}$ & Jordan & $\begin{array}{c}\text { Eastern } \\
\text { Mediterranean }\end{array}$ & 521 & 68 & $\begin{array}{l}\text { Lower middle } \\
\text { income }\end{array}$ & nurses/physicians & general hospital & l & self-administered & $75.00 \%$ & all & 6 \\
\hline $\begin{array}{c}\text { Teymourzadeh et al., } \\
2014 \text { [57] }\end{array}$ & Iran & $\begin{array}{c}\text { Eastern } \\
\text { Mediterranean }\end{array}$ & 301 & 35 & $\begin{array}{l}\text { Upper middle } \\
\text { income }\end{array}$ & nurses & general hospital & urban & self-administered & $73.00 \%$ & all & 8 \\
\hline $\begin{array}{l}\text { Abou-EIWafa et al., } \\
2014 \text { [58] }\end{array}$ & Egypt & $\begin{array}{c}\text { Eastern } \\
\text { Mediterranean }\end{array}$ & 275 & 51 & $\begin{array}{l}\text { Lower middle } \\
\text { income }\end{array}$ & nurses & $\begin{array}{c}\text { Emergency } \\
\text { department/internal } \\
\text { medicine }\end{array}$ & urban & self-administered & $96.15 \%$ & all & 8 \\
\hline $\begin{array}{l}\text { Alameddine et al., } 2015 \\
\text { [8] }\end{array}$ & Lebanon & $\begin{array}{c}\text { Eastern } \\
\text { Mediterranean }\end{array}$ & 572 & 48 & $\begin{array}{l}\text { Upper middle } \\
\text { income }\end{array}$ & nurses & health care setting & I & self-administered & $64.80 \%$ & random & 6 \\
\hline $\begin{array}{c}\text { Baran Aksakal et al., } \\
2015 \text { [59] }\end{array}$ & Turkey & European & 538 & 72 & $\begin{array}{l}\text { Upper middle } \\
\text { income }\end{array}$ & nurses & general hospital & 1 & $\begin{array}{l}\text { face-to-face } \\
\text { interview }\end{array}$ & $82.76 \%$ & all & 6 \\
\hline Baykan et al., 2015 [60] & Turkey & European & 597 & 151 & $\begin{array}{l}\text { Upper middle } \\
\text { income }\end{array}$ & physicians & $\begin{array}{l}\text { health care } \\
\text { workplace }\end{array}$ & l & self-administered & $75.90 \%$ & all & 8 \\
\hline Jiao et al., 2015 [61] & China & $\begin{array}{l}\text { Western } \\
\text { Pacific }\end{array}$ & 588 & 46 & $\begin{array}{l}\text { Upper middle } \\
\text { income }\end{array}$ & nurses & general hospital & urban & self-administered & $84.00 \%$ & random & 7 \\
\hline Park et al., 2015 [62] & Korea & $\begin{array}{l}\text { Western } \\
\text { Pacific }\end{array}$ & 970 & 243 & High income & nurses & general hospital & urban & self-administered & $95.20 \%$ & convenience & 8 \\
\hline Xing et al., 2015 [63] & China & $\begin{array}{l}\text { Western } \\
\text { Pacific }\end{array}$ & 840 & 90 & $\begin{array}{l}\text { Upper middle } \\
\text { income }\end{array}$ & nurses/physicians & primary care & rural & self-administered & $84.80 \%$ & purposive & 5 \\
\hline $\begin{array}{l}\text { Alkorashy et al., } 2016 \\
\text { [64] }\end{array}$ & Saudi Arabia & $\begin{array}{c}\text { Eastern } \\
\text { Mediterranean }\end{array}$ & 370 & 67 & High income & nurses & general hospital & urban & self-administered & $80.80 \%$ & convenience & 6 \\
\hline $\begin{array}{l}\text { Fallahi-Khoshknab } \\
\text { et al., 2016 [65] }\end{array}$ & Iran & $\begin{array}{c}\text { Eastern } \\
\text { Mediterranean }\end{array}$ & 5874 & 1187 & $\begin{array}{l}\text { Upper middle } \\
\text { income }\end{array}$ & $\begin{array}{l}\text { health care } \\
\text { professionals }\end{array}$ & general hospital & 1 & self-administered & $90.36 \%$ & random & 5 \\
\hline Jaradat et al., 2016 [9] & Palestine & $\begin{array}{c}\text { Eastern } \\
\text { Mediterranean }\end{array}$ & 343 & 13 & $\begin{array}{l}\text { Lower middle } \\
\text { income }\end{array}$ & nurses & $\begin{array}{l}\text { hospitals/primary } \\
\text { care }\end{array}$ & l & self-administered & $92.20 \%$ & l & 8 \\
\hline Quinn et al., 2016 [66] & U.S. & Americas & 1249 & 82 & High income & home care aides & home healthcare & 1 & self-administered & $44.20 \%$ & 1 & 6 \\
\hline Zafar et al., 2016 [7] & Pakistan & $\begin{array}{l}\text { Eastern } \\
\text { Mediterranean }\end{array}$ & 179 & 13 & $\begin{array}{l}\text { Lower middle } \\
\text { income }\end{array}$ & physicians & general hospital & urban & self-administered & $92.20 \%$ & all & 4 \\
\hline $\begin{array}{l}\text { Abdellah et al., } 2017 \\
\text { [67] }\end{array}$ & Egypt & $\begin{array}{c}\text { Eastern } \\
\text { Mediterranean }\end{array}$ & 134 & 19 & $\begin{array}{l}\text { Lower middle } \\
\text { income }\end{array}$ & $\begin{array}{l}\text { Health care } \\
\text { professionals }\end{array}$ & $\begin{array}{l}\text { emergency } \\
\text { department }\end{array}$ & urban & self-administered & $94.40 \%$ & l & 7 \\
\hline Boafo et al., 2016 [68] & Ghana & African & 592 & 44 & $\begin{array}{l}\text { Lower middle } \\
\text { income }\end{array}$ & nurses & general hospital & I & self-administered & $57.98 \%$ & random & 4 \\
\hline Cheung et al., 2017 [69] & China & $\begin{array}{l}\text { Western } \\
\text { Pacific }\end{array}$ & 720 & 113 & $\begin{array}{l}\text { Upper middle } \\
\text { income }\end{array}$ & nurses/physicians & general hospital & urban & self-administered & $80.00 \%$ & convenience & 7 \\
\hline
\end{tabular}


Table 1. Cont.

\begin{tabular}{|c|c|c|c|c|c|c|c|c|c|c|c|c|}
\hline Study & Country & WHO Region & Sample Size & Event & $\begin{array}{c}\text { Income } \\
\text { Classification }\end{array}$ & $\begin{array}{l}\text { Professional } \\
\text { Group }\end{array}$ & Setting & $\begin{array}{l}\text { Region of Health } \\
\text { Care Setting }\end{array}$ & $\begin{array}{l}\text { Method of Data } \\
\text { Collection }\end{array}$ & $\begin{array}{c}\text { Response } \\
\text { Rate }\end{array}$ & Sampling & $\begin{array}{l}\text { Quality } \\
\text { Score }\end{array}$ \\
\hline Jafree et al., 2017 [70] & Pakistan & $\begin{array}{c}\text { Eastern } \\
\text { Mediterranean }\end{array}$ & 309 & 98 & $\begin{array}{l}\text { Lower middle } \\
\text { income }\end{array}$ & nurses & general hospital & urban & self-administered & $34.80 \%$ & random & 6 \\
\hline Li et al., 2017 [71] & China & $\begin{array}{l}\text { Western } \\
\text { Pacific }\end{array}$ & 1932 & 206 & $\begin{array}{l}\text { Upper middle } \\
\text { income }\end{array}$ & nurses/physicians & pediatric hospital & urban & self-administered & $86.80 \%$ & random & 7 \\
\hline $\begin{array}{l}\text { Pekurinen et al., } 2017 \\
{[72]}\end{array}$ & Finland & European & 5228 & 1288 & High income & nurses & general hospital & I & self-administered & $70.00 \%$ & all & 7 \\
\hline $\begin{array}{c}\text { Ridenour et al., } 2017 \\
{[73]}\end{array}$ & U.S. & Americas & 309 & 118 & High income & nurses & general hospital & I & self-administered & $22.50 \%$ & random & 7 \\
\hline Shi et al., 2017 [6] & China & $\begin{array}{l}\text { Western } \\
\text { Pacific }\end{array}$ & 2796 & 335 & $\begin{array}{l}\text { Upper middle } \\
\text { income }\end{array}$ & $\begin{array}{l}\text { health care } \\
\text { professionals }\end{array}$ & general hospital & I & self-administered & $64.25 \%$ & convenience & 6 \\
\hline Sisawo et al., 2017 [74] & Gambia & African & 219 & 33 & low income & nurses & general hospital & mixed & $\begin{array}{l}\text { face-to-face } \\
\text { interview }\end{array}$ & $98.20 \%$ & purposive & 5 \\
\hline Chen et al., 2018 [75] & China & $\begin{array}{l}\text { Western } \\
\text { Pacific }\end{array}$ & 1831 & 111 & $\begin{array}{l}\text { Upper middle } \\
\text { income }\end{array}$ & nurses & general hospital & urban & self-administered & $92.30 \%$ & all & 5 \\
\hline Ifediora et al., 2018 [76] & Australia & $\begin{array}{l}\text { Western } \\
\text { Pacific }\end{array}$ & 168 & 6 & High income & physicians & primary care & I & self-administered & $56.00 \%$ & I & 7 \\
\hline $\begin{array}{c}\text { Olashore et al., } 2018 \\
\text { [77] }\end{array}$ & Botswana & African & 201 & 79 & $\begin{array}{l}\text { Upper middle } \\
\text { income }\end{array}$ & $\begin{array}{l}\text { health care } \\
\text { professionals }\end{array}$ & psychiatric & urban & self-administered & $95.70 \%$ & all & 3 \\
\hline Pandey et al., 2018 [78] & Nepal & $\begin{array}{l}\text { South-East } \\
\text { Asia }\end{array}$ & 200 & 22 & low income & nurses & general hospital & I & self-administered & $100.00 \%$ & random & 5 \\
\hline $\begin{array}{l}\text { Pihl-Thingvad et al., } \\
2018 \text { [15] }\end{array}$ & Denmark & European & 496 & 126 & High income & $\begin{array}{l}\text { health care } \\
\text { professionals }\end{array}$ & general hospital & urban & self-administered & $28.00 \%$ & all & 7 \\
\hline $\begin{array}{l}\text { Schablon et al., } 2018 \\
\text { [79] }\end{array}$ & Germany & European & 1984 & 1329 & High income & nurses & $\begin{array}{l}\text { hospital/elder } \\
\text { care/residential } \\
\text { facility }\end{array}$ & I & self-administered & $40.90 \%$ & random & 6 \\
\hline Yang et al., 2018 [80] & China & $\begin{array}{l}\text { Western } \\
\text { Pacific }\end{array}$ & 237 & 194 & $\begin{array}{l}\text { Upper middle } \\
\text { income }\end{array}$ & nurses & psychiatric & urban & self-administered & $84.50 \%$ & I & 5 \\
\hline Zhang et al., 2018 [81] & China & $\begin{array}{l}\text { Western } \\
\text { Pacific }\end{array}$ & 1024 & 149 & $\begin{array}{l}\text { Upper middle } \\
\text { income }\end{array}$ & nurses & general hospital & I & self-administered & $75.18 \%$ & snowball & 4 \\
\hline
\end{tabular}




\subsection{Pooled One-Year Prevalence of Workplace Physical Violence}

A total of 65 studies reported one-year prevalence of workplace physical violence against health care professionals perpetrated by patients or visitors, with prevalence estimates ranging from $2.75 \%$ to $88.31 \%$. The lowest one-year prevalence was found among nurses in Thailand [16] and the highest was found among psychiatric nurses in the United Kingdom [30]. The pooled one-year prevalence of workplace physical violence against health care professionals perpetrated by patients or visitors was $19.33 \%$ (95\% confidence interval (CI): $16.49-22.53 \%$, Figure 2$)$ by a random effects model. The analysis revealed significant heterogeneity between studies $\left(\mathrm{I}^{2}=98.8 \%, p<0.001\right)$.

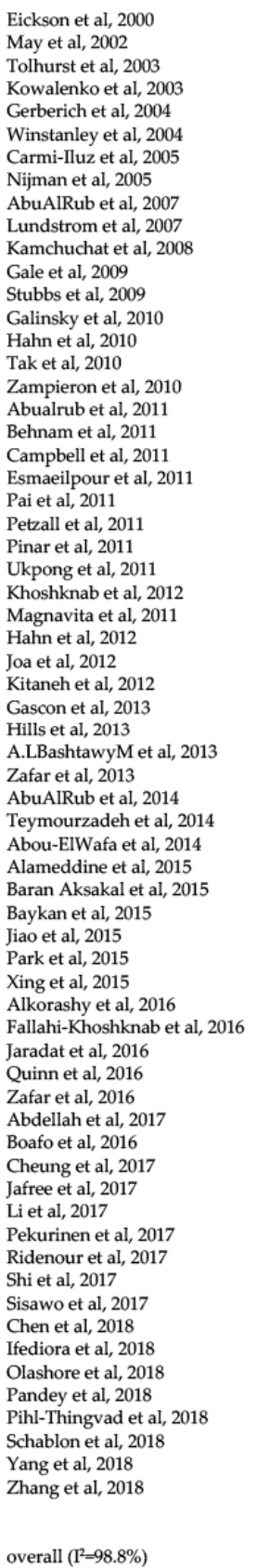

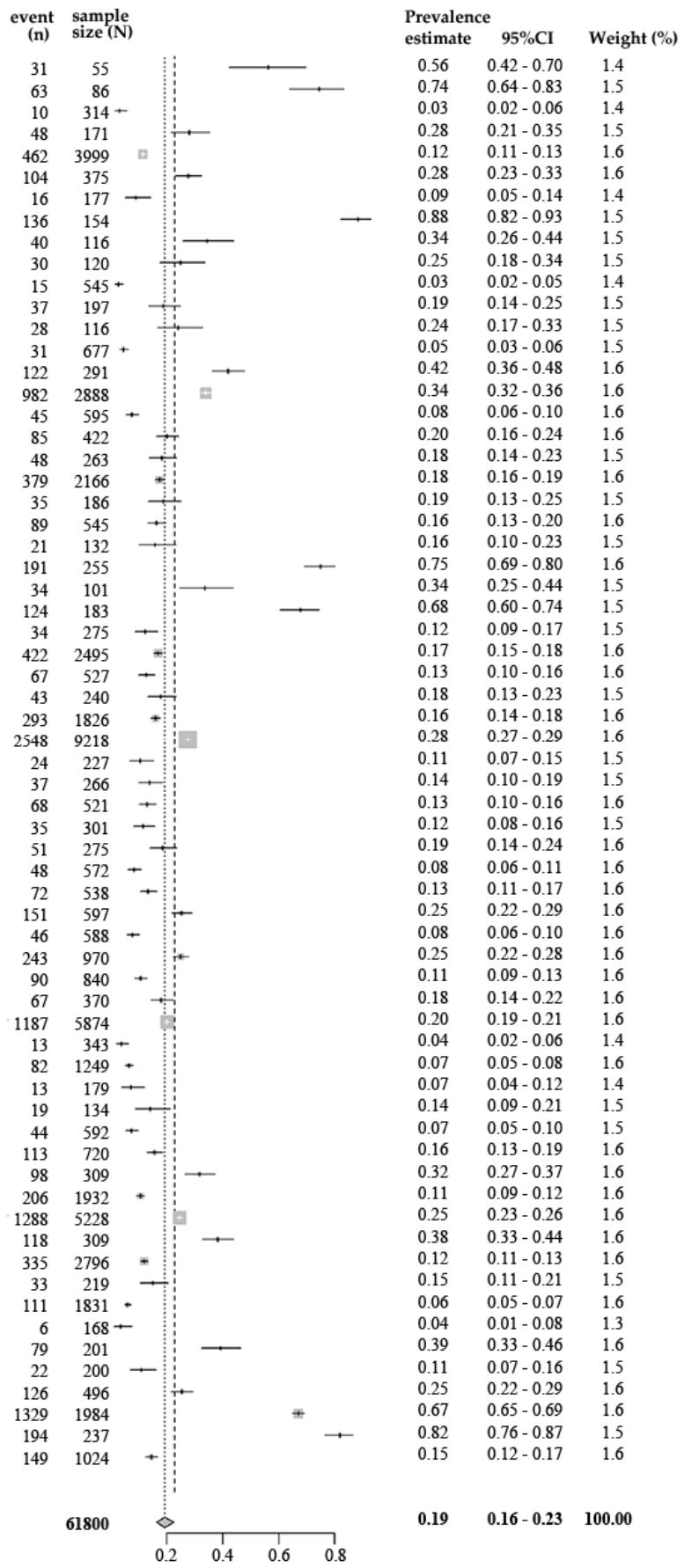

Figure 2. Forest plot of eligible studies. CI, confidence interval. 


\subsection{Subgroup Analyses}

For the regional level, pooled one-year prevalence of workplace physical violence against health care professionals perpetrated by patients or visitors was $26.38 \%$ (95\% CI: $18.42-36.25 \%$ ) in the European region, $23.61 \%$ (95\% CI: $15.25-34.67 \%$ ) in the Americas region, $20.71 \%$ (95\% CI: 8.59-42.07\%) in the African region, $17.07 \%$ (95\% CI: $13.15-21.86 \%$ ) in the eastern Mediterranean region, $14.53 \%$ (95\% CI: 10.05-20.54\%) in the Western Pacific region, and 5.62\% (95\% CI: 1.38-20.14\%) in the Southeast Asia region. The pooled one-year prevalence of workplace physical violence against health care professionals perpetrated by patients or visitors in high-income, upper-middle-income, lower-middle-income, and low-income countries was 21.66\% (95\% CI: 17.49-26.51\%), 19.98\% (95\% CI: 14.61-26.69\%), 13.75\% (95\% CI: $9.49-19.50 \%$ ), and $13.14 \%$ (95\% CI: 9.62-\%17.70\%), respectively.

Prevalence estimates varied by health care facilities and staff categories. The pooled one-year prevalence estimates in tertiary hospital, secondary hospital, primary care facilities, and nursing home were $22.48 \%$ (95\% CI: $15.35-31.69 \%$ ), $18.83 \%$ (95\% CI: 9.94-32.77\%), 6.51\% (95\% CI: $4.36-9.64 \%$ ), and $30.33 \%$ (95\% CI: $22.32-39.75 \%$ ), respectively. The pooled one-year prevalence of workplace physical violence against nurses perpetrated by patients or visitors was significantly higher than that against physicians $(22.99 \%$ vs. $14.66 \%, Q=4.38, p=0.0364)$. Studies conducted in rural and township areas had significantly lower prevalence estimates than urban areas $(6.11 \%$ vs. $26.16 \%, Q=7.93, p=0.0190)$. The pooled one-year prevalence of workplace physical violence against male health care professionals perpetrated by patients or visitors was similar to that against female health care professionals $(7.37 \%$ vs. $8.40 \%, Q=0.04, p=0.8392$ ).

Some methodological characteristics also influenced prevalence estimates across studies. When compared studies with sample size $>500$, studies with sample sizes $\leq 500$ had higher prevalence estimates $(13.96 \%$ vs. $24.48 \%, Q=9.91, p=0.0016)$. When compared studies with response rate $>50 \%$, studies with response rate $\leq 50 \%$ had higher prevalence estimates $(17.65 \%$ vs. $38.53 \%, Q=4.31$, $p=0.0379$ ). Subgroup analysis showed the sampling method, year of publication, and method for data collection were not statistically associated with prevalence estimates. All details about the subgroup analysis are provided in Table 2.

Table 2. Subgroup analysis of the pooled prevalence.

\begin{tabular}{|c|c|c|c|c|c|}
\hline \multirow[t]{2}{*}{ Subgroup } & \multirow[t]{2}{*}{ Studies } & \multirow{2}{*}{$\begin{array}{l}\text { Pooled Prevalence \% } \\
(95 \% \text { CI })\end{array}$} & \multirow[t]{2}{*}{$\mathrm{I}^{2}$} & \multicolumn{2}{|c|}{$\begin{array}{c}\text { Test of Difference within Each } \\
\text { Subgroup }\end{array}$} \\
\hline & & & & $Q$ & $p$ \\
\hline WHO Region & & & & 10.60 & 0.0599 \\
\hline European & 18 & $26.38(18.42-36.25)$ & $99.2 \%$ & & \\
\hline Americas & 10 & $23.61(15.25-34.67)$ & $99.0 \%$ & & \\
\hline African & 4 & $20.71(8.59-42.07)$ & $97.3 \%$ & & \\
\hline Eastern Mediterranean & 17 & $17.07(13.15-21.86)$ & $95.7 \%$ & & \\
\hline Western Pacific & 14 & $14.53(10.05-20.54)$ & $98.9 \%$ & & \\
\hline South-East Asia & 2 & $5.62(1.38-20.14)$ & $94.5 \%$ & & \\
\hline Income classification & & & & 9.84 & 0.0020 \\
\hline High-income & 32 & $21.66(17.49-26.51)$ & $99.0 \%$ & & \\
\hline Upper-middle-income & 20 & $19.98(14.61-26.69)$ & $98.7 \%$ & & \\
\hline Lower-middle-income & 11 & $13.75(9.49-19.50)$ & $93.6 \%$ & & \\
\hline Low-income & 2 & $13.14(9.62-17.70)$ & $33.6 \%$ & & \\
\hline Year of publication & & & & 1.06 & 0.3036 \\
\hline 2000-2010 & 17 & $22.83(15.31-32.61)$ & $98.6 \%$ & & \\
\hline 2011-2018 & 48 & $18.22(15.17-21.73)$ & $98.8 \%$ & & \\
\hline Sample size & & & & 9.91 & 0.0016 \\
\hline$\leq 500$ & 37 & $24.48(18.84-31.16)$ & $97.2 \%$ & & \\
\hline$>500$ & 28 & $13.96(10.99-17 \cdot 57)$ & $99.3 \%$ & & \\
\hline Response rate & & & & 4.31 & 0.0379 \\
\hline$\leq 50 \%$ & 7 & $38.53(18.75-63.00)$ & $99.4 \%$ & & \\
\hline$>50 \%$ & 58 & $17.65(15.33-20.23)$ & $98.1 \%$ & & \\
\hline
\end{tabular}


Table 2. Cont

\begin{tabular}{|c|c|c|c|c|c|}
\hline \multirow[t]{2}{*}{ Subgroup } & \multirow{2}{*}{ Studies } & \multirow{2}{*}{$\begin{array}{l}\text { Pooled Prevalence \% } \\
\text { (95\% CI) }\end{array}$} & \multirow{2}{*}{$\mathrm{I}^{2}$} & \multicolumn{2}{|c|}{$\begin{array}{c}\text { Test of Difference within Each } \\
\text { Subgroup }\end{array}$} \\
\hline & & & & $Q$ & $p$ \\
\hline Professional group & & & & 4.38 & 0.0364 \\
\hline nurses & 34 & $22.99(17.11-30.16)$ & $99.1 \%$ & & \\
\hline physicians & 10 & $14.66(10.67-19.81)$ & $94.4 \%$ & & \\
\hline Method of collection & & & & 0.88 & 0.6441 \\
\hline Self-administered & 59 & $19.66(16.60-23.14)$ & $98 \cdot 8 \%$ & & \\
\hline face-to-face interview & 4 & $13.93(6.39-27.76)$ & $96.3 \%$ & & \\
\hline Telephone interview & 2 & $21.61(7.40-48.73)$ & $98.8 \%$ & & \\
\hline Gender & & & & 0.04 & 0.8392 \\
\hline Male & 3 & 7.37 (2.00-23.69) & $89.5 \%$ & & \\
\hline Female & 3 & $8.40(6.72-10.46)$ & $37.9 \%$ & & \\
\hline Sampling & & & & 0.84 & 0.6572 \\
\hline all & 21 & $20.82(16.89-25.38)$ & $98.2 \%$ & & \\
\hline Random & 17 & $20.86(14.19-29.59)$ & $99.4 \%$ & & \\
\hline convenience & 17 & $17.64(12.82-23.79)$ & $97.1 \%$ & & \\
\hline $\begin{array}{l}\text { Region of health care } \\
\text { setting }\end{array}$ & & & & 7.93 & 0.0190 \\
\hline Urban & 27 & $26.16(19.11-34.69)$ & $98.5 \%$ & & \\
\hline Rural/township & 2 & $6.11(1.80-18.76)$ & $93.1 \%$ & & \\
\hline Mixed & 13 & $17.85(13.68-22.97)$ & $97.7 \%$ & & \\
\hline $\begin{array}{l}\text { Type of health care } \\
\text { setting }\end{array}$ & & & & 39.52 & $<0.0001$ \\
\hline Tertiary hospital & 18 & $22.48(15.35-31.69)$ & $98.4 \%$ & & \\
\hline Secondary hospital & 3 & $18 \cdot 83(9.94-32.77)$ & $91.3 \%$ & & \\
\hline Primary care facilities & 6 & $6.51(4.36-9.64)$ & $90.2 \%$ & & \\
\hline nursing home & 2 & $30.33(22.32-39.75)$ & $75.7 \%$ & & \\
\hline Quality score & & & & 3.92 & 0.0476 \\
\hline$\leq 5$ & 31 & $24.00(16.41-33.69)$ & $99.0 \%$ & & \\
\hline$>5$ & 34 & $15.86(13.53-18.51)$ & $98.3 \%$ & & \\
\hline
\end{tabular}

\subsection{Meta-Regression Analyses}

Bivariate meta-regression suggested higher prevalence estimates reported in studies with a smaller sample size $(\beta=-0.698, p=0.0098)$, in tertiary hospital $(\beta=1.470, p=0.0022)$, and lower quality score $(\beta=-0.213, p=0.0364)$. Specifically, sample size accounted for $8.72 \%$ of the heterogeneity, type of the health care setting accounted for $14.20 \%$ of the heterogeneity, and quality score accounted for $5.41 \%$ of the heterogeneity across studies. Finally, sample size, type of health care setting, and quality score were entered into multivariate meta-regression model. Of the multivariate model, type of health care setting $(\beta=1.835, p=0.0003)$ and quality score $(\beta=-0.301, p=0.0105)$ remained significant and accounted for $24.87 \%$ of the heterogeneity (Table 3 ). 
Table 3. Meta-regression analyses of the effects of potential moderators.

\begin{tabular}{|c|c|c|c|c|c|}
\hline \multirow{2}{*}{ Univariate Analysis } & \multirow{2}{*}{$\beta$} & \multicolumn{2}{|c|}{$95 \% \mathrm{CI}$} & \multirow{2}{*}{$\mathbf{R}^{2}$} & \multirow{2}{*}{$p$ Value } \\
\hline & & Lower & Upper & & \\
\hline Publish year (continuous variable) & -0.0483 & -0.1076 & 0.0109 & $2.29 \%$ & 0.1100 \\
\hline Sample size $(n<500$ vs. $n \geq 500)$ & -0.6983 & -1.2281 & -0.1685 & $8.72 \%$ & 0.0098 \\
\hline $\begin{array}{l}\text { Response rate (continuous } \\
\text { variable) }\end{array}$ & -0.7139 & -2.1540 & 0.7262 & $0.00 \%$ & 0.3313 \\
\hline $\begin{array}{l}\text { Income Classification (high } \\
\text { income vs. other) }\end{array}$ & 0.2798 & -0.2698 & 0.8294 & $0.00 \%$ & 0.3183 \\
\hline Professional (nurses vs. other) & -0.6344 & -1.5518 & 0.2831 & $1.66 \%$ & 0.1753 \\
\hline $\begin{array}{l}\text { Region of health care setting } \\
\text { (urban vs. rural) }\end{array}$ & 0.6527 & -0.0754 & 1.3808 & $4.78 \%$ & 0.0789 \\
\hline $\begin{array}{c}\text { Type of health care setting } \\
\text { (tertiary hospital vs. primary care } \\
\text { facilities) }\end{array}$ & 1.4696 & 0.5297 & 2.4095 & $14.20 \%$ & 0.0022 \\
\hline $\begin{array}{l}\text { Method of data collection } \\
\text { (Self-administered vs. others) }\end{array}$ & 0.4451 & -0.1229 & 1.0130 & $2.22 \%$ & 0.1245 \\
\hline $\begin{array}{c}\text { Quality score (continuous } \\
\text { variable) }\end{array}$ & -0.2125 & -0.4117 & -0.0134 & $5.41 \%$ & 0.0364 \\
\hline \multicolumn{6}{|l|}{ Multivariate Analysis } \\
\hline $\begin{array}{l}\text { Sample size }(n<500 \text { vs. } n \geq 500) \\
\text { Type of health care setting }\end{array}$ & -0.1671 & -0.7712 & 0.4369 & / & 0.5876 \\
\hline $\begin{array}{l}\text { (tertiary hospital vs. primary care } \\
\text { facilities) }\end{array}$ & 1.8345 & 0.8373 & 2.8316 & / & 0.0003 \\
\hline $\begin{array}{l}\text { Quality score (continuous } \\
\text { variable) }\end{array}$ & -0.3008 & -0.5314 & -0.0703 & l & 0.0105 \\
\hline Overall & & & & $24.87 \%$ & \\
\hline
\end{tabular}

\subsection{Sensitivity Analysis and Publication Bias}

After one-by-one removals of 65 studies, the pooled one-year prevalence of workplace physical violence against health care professionals perpetrated by patients or visitors varied from $18.55 \%(95 \%$ CI: $15.82-21.63 \%$ ) to $19.77 \%$ (95\% CI: $16.87-23.03 \%$ ), and the $\mathrm{I}^{2}$ statistic varied from $98.2 \%$ to $98.8 \%$. The results of the sensitivity analysis revealed that no individual study significantly influenced the results. Publication bias was not observed in this meta-analysis, with the $p$-value for the Begg's rank test being 0.1012 (Figure 3).

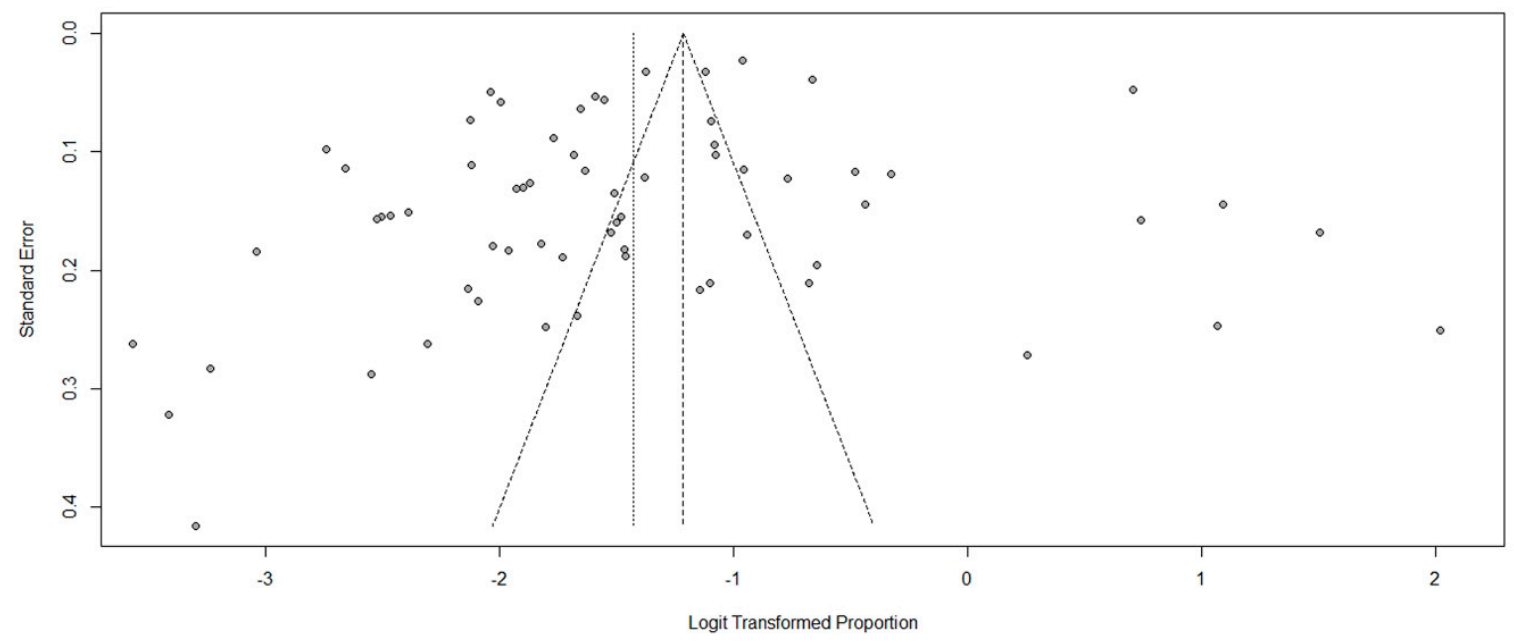

Figure 3. Funnel plots estimating small sample bias. 


\section{Discussion}

Using meta-analytical methods, we pooled the one-year prevalence estimates of workplace physical violence against health care professionals perpetrated by patients or visitors reported in 65 studies published between 2000 and 2018. Eligible studies included 61,800 health care professionals from 30 countries. The one-year prevalence of workplace physical violence against health care professionals perpetrated by patients or visitors was 19.33\% (95\% CI: 16.49-22.53\%) worldwide, or about one in five health care professionals annually. To the best of our knowledge, this study provided the first quantitative estimate of the prevalence of workplace physical violence against health care professionals perpetrated by patients or visitors worldwide.

Few review articles specifically focused on the prevalence of workplace physical violence against health care professionals perpetrated by patients or visitors. A systematic review conducted in 2008 found that, on average, $25 \%$ of health care professionals have experienced workplace physical violence perpetrated by patients or visitors in general hospital [82]. Another systematic review conducted in 2013 found that $2 \%$ to $32 \%$ hospital workers have experienced workplace physical violence perpetrated by patients or visitors [18]. Previous systematic review did not synthesize results by meta-analysis. This current meta-analysis revealed that $19.33 \%$ of health care professionals have experienced workplace violence perpetrated by patients or visitors worldwide annually. Our estimate of $19.33 \%$ was pooled based on 65 studies across all health care sectors, diverse health care professional types, and multiple countries. Prevalence estimates varied by region, with $26.38 \%$ in the European region, $23.61 \%$ in the Americas region, $20.71 \%$ in the African region, $17.07 \%$ in the eastern Mediterranean region, $14.53 \%$ in the western Pacific region, and 5.62\% in the Southeast Asia region. Though eligible studies covered all WHO regions, prevalence studies were sparse in the Southeast Asia and African region. Besides, half of the eligible studies in this meta-analysis were conducted in high-income countries. More studies in low-income and lower-middle-income countries were needed.

Among eligible studies, more than half of the studies were published after 2010. We found that the year of publication was not associated with the prevalence estimates. Administrative strategies, preventive interventions, and policy against workplace violence have been advocated in health sectors over the decade $[83,84]$. A survey conducted by the National Crime Victimization suggested that the rate of nonfatal workplace violence has declined by 35\% in the United States from 2002 to 2009 [3]. In the present study, the one-year prevalence estimate was not significantly declined worldwide based on the result of the subgroup analysis. Practical intervention in health care sectors was still an urgent need. Our finding may vary with geographical location because each country had its own special working environment and conditions. Future research could benefit from examining the national time trend of workplace physical violence and exploring how country-specific social factors and policy affected it.

The results revealed that studies with sample sizes $\leq 500$ and studies with low response rate had significantly higher one-year prevalence estimates. The studies' characteristics obviously influenced prevalence estimates of workplace physical. Studies with fewer participants generally yielded more extreme prevalence estimates [85], which may be attributed to selection bias and publication bias. Studies with a low response rate provided higher prevalence estimates as a result of report bias. In a meta-analysis of elder abuse, the result also suggested studies with small sample sizes were more likely to produce higher prevalence estimates [86].

The result of the subgroup analysis suggested that nurses experienced more workplace physical violence perpetrated by patients or visitors than physicians. This phenomenon was supported by numerous epidemiological studies $[28,69,87,88]$. Another meta-analysis also emphasized the disparate workplace physical violence experiences in nurses and physicians [22]. The working content and duties were quite different between nurses and physicians, as well as nurse-patient interaction and physician-patient interaction [69]. Nurses experiencing more physical violence may account for their gender, occupational prestige, and closer contact with patients and visitors [88]. Besides, as physicians dominated the process of treatment, patients or visitors might show more obedience and respect to physicians. We noticed that most of studies were specifically assessed workplace physical violence 
against nurses, while evidence of physicians was relatively limited. If more evidence of workplace physical violence against physicians was available, the finding of professional imparity might be more credible.

Gender difference was not observed in this meta-analysis. In a systematic review emphasizing gender difference of physical violence, the researcher found that numerous studies showed male health care professionals experienced more workplace physical violence than females [89]. This systematic review also suggested that 19 studies revealed a non-significant association between workplace physical violence and gender [89]. In this meta-analysis, only three eligible studies reported rates of workplace physical violence perpetrated by patients or visitors for male and female health care professionals separately, which yielded a very limited result. Gender difference of workplace physical violence against health care professionals was an undetermined issue. It is necessary for future research to provide gender-specific prevalence estimates. Those studies could help us understand demographic characteristic of victims and provide evidences for well-targeted intervention.

Subgroup analyses revealed that health care professionals working in nursing homes experienced more physical violence from patients or visitors than those in other health care settings. Patients with dementia or disability in nursing homes might present more aggressive behavior and physical violence against health care professionals than general patients [37]. Except nursing homes, health care professionals working in tertiary hospitals experienced more workplace physical violence than those working in primary care facilities. To date, only a handful studies compared workplace physical violence between different health care settings. Gascon et al. found that health workers in a large hospital experience more physical violence than those in a small hospital and primary health center [52]. The risk factors of workplace physical violence such as overcrowding, noisy, long waiting time, and short consultation time occurred more frequently in tertiary hospitals $[13,53,74,90]$. Additionally, medical conditions of patients were generally severer in tertiary hospitals than those in primary care facilities. However, patients had higher expectations in tertiary hospitals than in primary care facilities $[90,91]$. Thus, patients might show less satisfaction and more aggression in tertiary hospitals. Clearly, the scant evidence suggested a need for further research exploring the role of health care settings.

Health care professionals working in rural or township areas experienced less workplace physical violence perpetrated by patients or visitors than those in urban areas. Few studies had emphasized the disparity between urban and rural areas [74]. Patients with a severe condition and high demand were prone to seek help directly in a tertiary hospital located in urban areas [92]. Thus, health care professionals were faced with more stressful working environment in urban areas, which increased the risk of workplace violence [13]. Research of violence also suggested that urban-rural disparity may be explained by social factors such as inequality and poor social cohesion [11]. Here, only two eligible studies specifically evaluated the prevalence of workplace physical violence against health care professionals perpetrated by patients or visitors in a rural or township area. More studies were needed to obtain a reliable estimate.

There were several limitations in the present study. First, although our study included relevant studies across 30 countries, half of the eligible studies were from high-income countries. Prevalence studies were scarce for many countries, especially for lower-middle-income and low-income countries. Considering the inconsistency of the health care environment and working conditions across the world, more prevalence studies in low-income and lower-middle-income countries are needed to understand the panorama of workplace physical violence against health care professionals. Second, the ability to compare findings and understand the magnitude of pooled prevalence was severely hampered by inconsistent methodology between studies, including inconsistent definitions, response rate, and methods of data collection. Although we have excluded those studies without description of definition and measurement, inconsistency was still inescapable. Numerous studies adopted self-designed and self-administrated questionnaire to measure workplace physical violence. It is hard to compare findings without a standard assessment tool. Thus, future research should develop a standard and comprehensive used assessment tool to measure workplace physical violence. 


\section{Conclusions}

The pooled one-year prevalence of workplace physical violence against health care professionals perpetrated by patients or visitors was 19.33\% (95\% CI: 16.49-22.53\%). About one in five health care professionals experienced workplace physical violence perpetrated by patients or visitors annually. One-year prevalence estimates varied significantly regarding the country of study, sample size, response rate, professional group, region of health care setting, and type of health care setting. Significant moderators for heterogeneity included sample size, type of health care setting, and quality score. Future research can benefit from exploring gender differences, occupational differences, and time trends in workplace physical violence against health care professionals. More practical intervention and policy defensed workplace physical violence were needed to ensure the safety of health care professionals.

Supplementary Materials: The following are available online at http://www.mdpi.com/1660-4601/17/1/299/s1, Table S1: PRISMA-2009-checklist; Table S2: Database searches results; Table S3: Methodological quality assessment of the eligible studies.

Author Contributions: Y.-L.L., R.-Q.L., D.Q., and S.-Y.X. designed the study. All authors oversaw its implementation. Y.-L.L. and R.-Q.L. searched the literature, selected studies, extracted data, and conducted quality assessment. Y.-L.L. and D.Q. planned the analyses and did the meta-analyses and meta-regressions. Y.-L.L. drafted the manuscript. S.-Y.X. and D.Q. contributed writing to subsequent versions of the manuscript. All authors reviewed the study findings and read and approved the final version before submission. All authors have read and agreed to the published version of the manuscript.

Funding: This research was supported by the Ministry of Science and Technology of China (Grant NO: 2016YFC0900802).

Conflicts of Interest: We declare no competing interest.

\section{References}

1. Phillips, J.P. Workplace Violence against Health Care Workers in the United States. N. Engl. J. Med. 2016, 374, 1661-1669. [CrossRef] [PubMed]

2. Piquero, N.L.; Piquero, A.R.; Craig, J.M.; Clipper, S.J. Assessing research on workplace violence, $2000-2012$. Aggress. Violent Behav. 2013, 18, 383-394. [CrossRef]

3. Harrell, E. Workplace Violence, 1993-2009. National Crime Victimization Survey and the Census of Fatal Occupational Injuries; Bureau of Justice Statistics: Washington, DC, USA, 2011.

4. Wiskow, C. Guidelines on Workplace Violence in the Health Sector. Available online: https://www.who. int/violence_injury_prevention/violence/interpersonal/en/WV_ComparisonGuidelines.pdf (accessed on 21 March 2019).

5. Lanctot, N.; Guay, S. The aftermath of workplace violence among healthcare workers: A systematic literature review of the consequences. Aggress. Violent Behav. 2014, 19, 492-501. [CrossRef]

6. Shi, L.; Wang, L.; Jia, X.; Li, Z.; Mu, H.; Liu, X.; Peng, B.; Li, A.; Fan, L. Prevalence and correlates of symptoms of post-traumatic stress disorder among Chinese healthcare workers exposed to physical violence: A cross-sectional study. BMJ Open 2017, 7, e016810. [CrossRef]

7. Zafar, W.; Khan, U.R.; Siddiqui, S.A.; Jamali, S.; Razzak, J.A. Workplace Violence and Self-reported Psychological Health: Coping with Post-traumatic Stress, Mental Distress, and Burnout among Physicians Working in the Emergency Departments Compared to Other Specialties in Pakistan. J. Emerg. Med. 2016, 50, 167-177. [CrossRef]

8. Alameddine, M.; Mourad, Y.; Dimassi, H. A National Study on Nurses' Exposure to Occupational Violence in Lebanon: Prevalence, Consequences and Associated Factors. PLoS ONE 2015, 10, e0137105. [CrossRef]

9. Jaradat, Y.; Nielsen, M.B.; Kristensen, P.; Nijem, K.; Bjertness, E.; Stigum, H.; Bast-Pettersen, R. Workplace aggression, psychological distress, and job satisfaction among Palestinian nurses: A cross-sectional study. Appl. Nurs. Res. ANR 2016, 32, 190-198. [CrossRef]

10. McGovern, P.; Kochevar, L.; Lohman, W.; Zaidman, B.; Gerberich, S.G.; Nyman, J.; Findorff-Dennis, M. The cost of work-related physical assaults in Minnesota. Health Serv. Res. 2000, 35, 663.

11. Sumner, S.A.; Mercy, J.A.; Dahlberg, L.L.; Hillis, S.D.; Klevens, J.; Houry, D. Violence in the United States: Status, Challenges, and Opportunities. JAMA 2015, 314, 478-488. [CrossRef] 
12. Schat, A.C.H.; Kelloway, E.K. Reducing the adverse consequences of workplace aggression and violence: The buffering effects of organizational support. J. Occup. Health Psychol. 2003, 8, 110-122. [CrossRef]

13. Nowrouzi-Kia, B.; Isidro, R.; Chai, E.; Usuba, K.; Chen, A. Antecedent factors in different types of workplace violence against nurses: A systematic review. Aggress. Violent Behav. 2019, 44, 1-7. [CrossRef]

14. Guay, S.; Goncalves, J.; Jarvis, J. Verbal violence in the workplace according to victims' sex a systematic review of the literature. Aggress. Violent Behav. 2014, 19, 572-578. [CrossRef]

15. Pihl-Thingvad, J.; Brandt, L.P.A.; Andersen, L.L. Consistent Use of Assistive Devices for Patient Transfer Is Associated with Less Patient-Initiated Violence: Cross-Sectional Study Among Health Care Workers at General Hospitals. Workplace Health Saf. 2018, 66, 453-461. [CrossRef] [PubMed]

16. Kamchuchat, C.; Chongsuvivatwong, V.; Oncheunjit, S.; Yip, T.W.; Sangthong, R. Workplace violence directed at nursing staff at a general hospital in Southern Thailand. J. Occup. Health 2008, 50, 201-207. [CrossRef]

17. May, D.D.; Grubbs, L.M. The extent, nature, and precipitating factors of nurse assault among three groups of registered nurses in a regional medical center. J. Emerg. Nurs. JEN Off. Publ. Emerg. Dep. Nurses Assoc. 2002, 28, 11-17. [CrossRef]

18. Nikathil, S.; Olaussen, A.; Gocentas, R.A.; Symons, E.; Mitra, B. Review article: Workplace violence in the emergency department: A systematic review and meta analysis. Emerg. Med. Australas. 2017, 29, 265-275. [CrossRef]

19. Cornaggia, C.M.; Beghi, M.; Pavone, F.; Barale, F. Aggression in psychiatry wards: A systematic review. Psychiatry Res. 2011, 189, 10-20. [CrossRef]

20. Taylor, J.L.; Rew, L. A systematic review of the literature: Workplace violence in the emergency department. J. Clin. Nurs. 2011, 20, 1072-1085. [CrossRef]

21. Spector, P.E.; Zhou, Z.E.; Che, X.X. Nurse exposure to physical and nonphysical violence, bullying, and sexual harassment: A quantitative review. Int. J. Nurs. Stud. 2014, 51, 72-84. [CrossRef]

22. Lu, L.; Dong, M.; Wang, S.B.; Zhang, L.; Ng, C.H.; Ungvari, G.S.; Li, J.; Xiang, Y.T. Prevalence of Workplace Violence Against Health-Care Professionals in China: A Comprehensive Meta-Analysis of Observational Surveys. Trauma Violence Abus. 2018. [CrossRef]

23. Pompeii, L.; Dement, J.; Schoenfisch, A.; Lavery, A.; Souder, M.; Smith, C.; Lipscomb, H. Perpetrator, worker and workplace characteristics associated with patient and visitor perpetrated violence (Type II) on hospital workers: A review of the literature and existing occupational injury data. J. Saf. Res. 2013, 44, 57-64. [CrossRef] [PubMed]

24. Erickson, L.; Williams-Evans, S.A. Attitudes of emergency nurses regarding patient assaults. J. Emerg. Nurs. JEN Off. Publ. Emerg. Dep. Nurses Assoc. 2000, 26, 210-215. [CrossRef]

25. Tolhurst, H.; Baker, L.; Murray, G.; Bell, P.; Sutton, A.; Dean, S. Rural general practitioner experience of work-related violence in Australia. Aust. J. Rural Health 2003, 11, 231-236. [PubMed]

26. Kowalenko, T.; Walters, B.L.; Compton, S. Workplace violence: A survey of Michigan College of Emergency Physicians. Ann. Emerg. Med. 2003, 42, S6.

27. Gerberich, S.G.; Church, T.R.; McGovern, P.M.; Hansen, H.E.; Nachreiner, N.M.; Geisser, M.S.; Ryan, A.D.; Mongin, S.J.; Watt, G.D. An epidemiological study of the magnitude and consequences of work related violence: The Minnesota Nurses' Study. Occup. Environ. Med. 2004, 61, 495-503. [CrossRef] [PubMed]

28. Winstanley, S.; Whittington, R. Aggression towards health care staff in a UK general hospital: Variation among professions and departments. J. Clin. Nurs. 2004, 13, 3-10. [CrossRef]

29. Carmi-Iluz, T.; Peleg, R.; Freud, T.; Shvartzman, P. Verbal and physical violence towards hospital- and community-based physicians in the Negev: An observational study. BMC Health Serv. Res. 2005, 5, 54. [CrossRef]

30. Nijman, H.; Bowers, L.; Oud, N.; Jansen, G. Psychiatric nurses' experiences with inpatient aggression. Aggress. Behav. 2005, 31, 217-227. [CrossRef]

31. AbuAlRub, R.F.; Khalifa, M.F.; Habbib, M.B. Workplace violence among Iraqi hospital nurses. J. Nurs. Scholarsh. Off. Publ. Sigma Theta Tau Int. Honor Soc. Nurs. 2007, 39, 281-288. [CrossRef]

32. Lundstrom, M.; Saveman, B.I.; Eisemann, M.; Astrom, S. Prevalence of violence and its relation to caregivers' demographics and emotional reactions: An explorative study of caregivers working in group homes for persons with learning disabilities. Scand. J. Caring Sci. 2007, 21, 84-90. [CrossRef]

33. Gale, C.; Arroll, B.; Coverdale, J. The 12-Month Prevalence of Patient-Initiated Aggression against Psychiatrists: A New Zealand National Survey. Int. J. Psychiatry Med. 2009, 39, 79-87. [CrossRef] [PubMed] 
34. Stubbs, B.; Dickens, G. Physical assault by patients against physiotherapists working in mental health settings. Physiotherapy 2009, 95, 170-175. [CrossRef] [PubMed]

35. Galinsky, T.; Feng, H.A.; Streit, J.; Brightwell, W.; Pierson, K.; Parsons, K.; Proctor, C. Risk Factors Associated with Patient Assaults of Home Healthcare Workers. Rehabil. Nurs. 2010, 35, 206-215. [CrossRef] [PubMed]

36. Hahn, S.; Müller, M.; Needham, I.; Dassen, T.; Kok, G.; Halfens, R.J.G. Factors associated with patient and visitor violence experienced by nurses in general hospitals in Switzerland: A cross-sectional survey. J. Clin. Nurs. 2010, 19, 3535-3546. [CrossRef] [PubMed]

37. Tak, S.; Sweeney, M.H.; Alterman, T.; Baron, S.; Calvert, G.M. Workplace Assaults on Nursing Assistants in US Nursing Homes: A Multilevel Analysis. Am. J. Public Health 2010, 100, 1938-1945. [CrossRef]

38. Zampieron, A.; Galeazzo, M.; Turra, S.; Buja, A. Perceived aggression towards nurses: Study in two Italian health institutions. J. Clin. Nurs. 2010, 19, 2329-2341. [CrossRef]

39. Abualrub, R.F.; Al-Asmar, A.H. Physical violence in the workplace among Jordanian hospital nurses. J. Transcult. Nurs. Off. J. Transcult. Nurs. Soc. 2011, 22, 157-165. [CrossRef]

40. Behnam, M.; Tillotson, R.D.; Davis, S.M.; Hobbs, G.R. Violence in the emergency department: A national survey of emergency medicine residents and attending physicians. J. Emerg. Med. 2011, 40, 565-579. [CrossRef]

41. Campbell, J.C.; Messing, J.T.; Kub, J.; Agnew, J.; Fitzgerald, S.; Fowler, B.; Sheridan, D.; Lindauer, C.; Deaton, J.; Bolyard, R. Workplace violence: Prevalence and risk factors in the safe at work study. J. Occup. Environ. Med. 2011, 53, 82-89. [CrossRef]

42. Esmaeilpour, M.; Salsali, M.; Ahmadi, F. Workplace violence against Iranian nurses working in emergency departments. Int. Nurs. Rev. 2011, 58, 130-137. [CrossRef]

43. Pai, H.C.; Lee, S. Risk factors for workplace violence in clinical registered nurses in Taiwan. J. Clin. Nurs. 2011, 20, 1405-1412. [CrossRef] [PubMed]

44. Petzall, K.; Tallberg, J.; Lundin, T.; Suserud, B.O. Threats and violence in the Swedish pre-hospital emergency care. Int. Emerg. Nurs. 2011, 19, 5-11. [CrossRef] [PubMed]

45. Pinar, R.; Ucmak, F. Verbal and physical violence in emergency departments: A survey of nurses in Istanbul, Turkey. J. Clin. Nurs. 2011, 20, 510-517. [CrossRef] [PubMed]

46. Ukpong, D.I.; Owoeye, O.; Udofia, O.; Abasiubong, F.; Ukpong, S. Violence against mental health staff: A survey in a Nigerian psychiatric hospital. Psychiatrist 2011, 35, 46-49. [CrossRef]

47. Khoshknab, M.F.; Tamizi, Z.; Ghazanfari, N.; Mehrabani, G. Prevalence of workplace violence in psychiatric wards, Tehran, Iran. Pak. J. Biol. Sci. PJBS 2012, 15, 680-684. [CrossRef]

48. Magnavita, N.; Heponiemi, T. Workplace violence against nursing students and nurses: An Italian experience. J. Nurs. Scholarsh. Off. Publ. Sigma Theta Tau Int. Honor Soc. Nurs. 2011, 43, 203-210. [CrossRef]

49. Hahn, S.; Hantikainen, V.; Needham, I.; Kok, G.; Dassen, T.; Halfens, R.J.G. Patient and visitor violence in the general hospital, occurrence, staff interventions and consequences: A cross-sectional survey. J. Adv. Nurs. 2012, 68, 2685-2699. [CrossRef]

50. Joa, T.S.; Morken, T. Violence towards personnel in out-of-hours primary care: A cross-sectional study. Scand. J. Prim. Health Care 2012, 30, 55-60. [CrossRef]

51. Kitaneh, M.; Hamdan, M. Workplace violence against physicians and nurses in Palestinian public hospitals: A cross-sectional study. BMC Health Serv. Res. 2012, 12, 469. [CrossRef]

52. Gascon, S.; Leiter, M.P.; Andrés, E.; Santed, M.A.; Pereira, J.P.; Cunha, M.J.; Albesa, A.; Montero-Marín, J.; García-Campayo, J.; Martínez-Jarreta, B. The role of aggressions suffered by healthcare workers as predictors of burnout. J. Clin. Nurs. 2013, 22, 3120-3129. [CrossRef]

53. Hills, D.J.; Joyce, C.M. Personal, professional, and work factors associated with Australian clinical medical practitioners' experiences of workplace aggression. Ann. Occup. Hyg. 2013, 57, 898-912. [PubMed]

54. Albashtawy, M. Workplace violence against nurses in emergency departments in Jordan. Int. Nurs. Rev. 2013, 60, 550-555. [CrossRef] [PubMed]

55. Zafar, W.; Siddiqui, E.; Ejaz, K.; Shehzad, M.U.; Khan, U.R.; Jamali, S.; Razzak, J.A. Health care personnel and workplace violence in the emergency departments of a volatile metropolis: Results from Karachi, Pakistan. J. Emerg. Med. 2013, 45, 761-772. [CrossRef] [PubMed]

56. AbuAlRub, R.F.; Al Khawaldeh, A.T. Workplace physical violence among hospital nurses and physicians in underserved areas in Jordan. J. Clin. Nurs. 2014, 23, 1937-1947. [CrossRef] [PubMed] 
57. Teymourzadeh, E.; Rashidian, A.; Arab, M.; Akbari-Sari, A.; Hakimzadeh, S.M. Nurses exposure to workplace violence in a large teaching hospital in Iran. Int. J. Health Policy Manag. 2014, 3, 301-305. [CrossRef]

58. Abou-ElWafa, H.S.; El-Gilany, A.H.; Abd-El-Raouf, S.E.; Abd-Elmouty, S.M.; El-Sayed, R.E.S.H. Workplace Violence Against Emergency Versus Non-Emergency Nurses in Mansoura University Hospitals, Egypt. J. Interpers. Violence 2015, 30, 857-872. [CrossRef]

59. Baran Aksakal, F.N.; Karaşahin, E.F.; Uğraş Dikmen, A.; Avci, E.; Özkan, S. Workplace physical violence, verbal violence, and mobbing experienced by nurses at a university hospital. Turk. J. Med Sci. 2015, 45, 1360-1368. [CrossRef]

60. Baykan, Z.; Oktem, I.S.; Cetinkaya, F.; Nacar, M. Physician exposure to violence: A study performed in Turkey. Int. J. Occup. Saf. Ergon. 2015, 21, 291-297. [CrossRef]

61. Jiao, M.; Ning, N.; Li, Y.; Gao, L.; Cui, Y.; Sun, H.; Kang, Z.; Liang, L.; Wu, Q.; Hao, Y. Workplace violence against nurses in Chinese hospitals: A cross-sectional survey. BMJ Open 2015, 5, e006719. [CrossRef]

62. Park, M.; Cho, S.H.; Hong, H.J. Prevalence and Perpetrators of Workplace Violence by Nursing Unit and the Relationship Between Violence and the Perceived Work Environment. J. Nurs. Scholarsh. 2015, 47, 87-95. [CrossRef]

63. Xing, K.; Jiao, M.; Ma, H.; Qiao, H.; Hao, Y.; Li, Y.; Gao, L.; Sun, H.; Kang, Z.; Liang, L.; et al. Physical Violence against General Practitioners and Nurses in Chinese Township Hospitals: A Cross-Sectional Survey. PLoS ONE 2015, 10, e0142954. [CrossRef] [PubMed]

64. Alkorashy, H.A.E.; Al Moalad, F.B. Workplace violence against nursing staff in a Saudi university hospital. Int. Nurs. Rev. 2016, 63, 226-232. [CrossRef] [PubMed]

65. Fallahi-Khoshknab, M.; Oskouie, F.; Najafi, F.; Ghazanfari, N.; Tamizi, Z.; Afshani, S. Physical violence against health care workers: A nationwide study from Iran. Iran. J. Nurs. Midwifery Res. 2016, 21, 232-238. [CrossRef] [PubMed]

66. Quinn, M.M.; Markkanen, P.K.; Galligan, C.J.; Sama, S.R.; Kriebel, D.; Gore, R.J.; Brouillette, N.M.; Okyere, D.; Sun, C.; Punnett, L.; et al. Occupational health of home care aides: Results of the safe home care survey. Occup. Environ. Med. 2016, 73, 237-245. [CrossRef] [PubMed]

67. Abdellah, R.F.; Salama, K.M. Prevalence and risk factors of workplace violence against health care workers in emergency department in Ismailia, Egypt. Pan Afr. Med. J. 2017, 26, 21. [CrossRef] [PubMed]

68. Boafo, I.M.; Hancock, P. Workplace Violence Against Nurses: A Cross-Sectional Descriptive Study of Ghanaian Nurses. Sage Open 2017, 7. [CrossRef]

69. Cheung, T.; Lee, P.H.; Yip, P.S.F. Workplace Violence toward Physicians and Nurses: Prevalence and Correlates in Macau. Int. J. Environ. Res. Public Health 2017, 14, 879. [CrossRef]

70. Jafree, S.R. Workplace violence against women nurses working in two public sector hospitals of Lahore, Pakistan. Nurs. Outlook 2017, 65, 420-427. [CrossRef]

71. Li, Z.; Yan, C.M.; Shi, L.; Mu, H.T.; Li, X.; Li, A.Q.; Zhao, C.S.; Sun, T.; Gao, L.; Fan, L.H.; et al. Workplace violence against medical staff of Chinese children's hospitals: A cross-sectional study. PLoS ONE 2017, 12, e0179373. [CrossRef]

72. Pekurinen, V.; Willman, L.; Virtanen, M.; Kivimaki, M.; Vahtera, J.; Valimaki, M. Patient Aggression and the Wellbeing of Nurses: A Cross-Sectional Survey Study in Psychiatric and Non-Psychiatric Settings. Int. J. Environ. Res. Public Health 2017, 14, 1245. [CrossRef]

73. Ridenour, M.L.; Hendricks, S.; Hartley, D.; Blando, J.D. Workplace Violence and Training Required by New Legislation Among NJ Nurses. J. Occup. Environ. Med. 2017, 59, e35-e40. [CrossRef] [PubMed]

74. Sisawo, E.J.; Ouédraogo, S.Y.Y.A.; Huang, S.L. Workplace violence against nurses in the Gambia: Mixed methods design. BMC Health Serv. Res. 2017, 17, 311. [CrossRef] [PubMed]

75. Chen, X.; Lv, M.; Wang, M.; Wang, X.; Liu, J.; Zheng, N.; Liu, C. Incidence and risk factors of workplace violence against nurses in a Chinese top-level teaching hospital: A cross-sectional study. Appl. Nurs. Res. ANR 2018, 40, 122-128. [CrossRef] [PubMed]

76. Ifediora, C.O. Evaluation of aggression in Australian after-hours doctor home-visit services. J. Eval. Clin. Pract. 2018, 24, 388-395. [CrossRef]

77. Olashore, A.A.; Akanni, O.O.; Molebatsi, K.; Ogunjumo, J.A. Post-traumatic stress disorder among the staff of a mental health hospital: Prevalence and risk factors. S. Afr. J. Psychiatry 2018, 24. [CrossRef]

78. Pandey, M.; Bhandari, T.R.; Dangal, G. Workplace Violence and its Associated Factors among Nurses. J. Nepal Health Res. Counc. 2018, 15, 235-241. [CrossRef] 
79. Schablon, A.; Wendeler, D.; Kozak, A.; Nienhaus, A.; Steinke, S. Prevalence and consequences of aggression and violence towards nursing and care staff in Germany-A survey. Int. J. Environ. Res. Public Health 2018, 15, 1274. [CrossRef]

80. Yang, B.X.; Stone, T.E.; Petrini, M.A.; Morris, D.L. Incidence, Type, Related Factors, and Effect of Workplace Violence on Mental Health Nurses: A Cross-sectional Survey. Arch. Psychiatr. Nurs. 2018, 32, 31-38. [CrossRef]

81. Zhang, S.E.; Liu, W.; Wang, J.; Shi, Y.; Xie, F.; Cang, S.; Sun, T.; Fan, L. Impact of workplace violence and compassionate behaviour in hospitals on stress, sleep quality and subjective health status among Chinese nurses: A cross-sectional survey. BMJ Open 2018, 8, e019373. [CrossRef]

82. Hahn, S.; Zeller, A.; Needham, I.; Kok, G.; Dassen, T.; Halfens, R.J.G. Patient and visitor violence in general hospitals: A systematic review of the literature. Aggress. Violent Behav. 2008, 13, 431-441. [CrossRef]

83. Hills, D.; Joyce, C. A review of research on the prevalence, antecedents, consequences and prevention of workplace aggression in clinical medical practice. Aggress. Violent Behav. 2013, 18, 554-569. [CrossRef]

84. Wassell, J.T. Workplace violence intervention effectiveness: A systematic literature review. Saf. Sci. 2009, 47, 1049-1055. [CrossRef]

85. Mata, D.A.; Ramos, M.A.; Bansal, N.; Khan, R.; Guille, C.; Di Angelantonio, E.; Sen, S. Prevalence of Depression and Depressive Symptoms Among Resident Physicians: A Systematic Review and Meta-analysis. JAMA 2015, 314, 2373-2383. [CrossRef] [PubMed]

86. Yon, Y.; Mikton, C.R.; Gassoumis, Z.D.; Wilber, K.H. Elder abuse prevalence in community settings: A systematic review and meta-analysis. Lancet Glob. Health 2017, 5, e147-e156. [CrossRef]

87. Lepping, P.; Lanka, S.V.N.; Turner, J.; Stanaway, S.E.R.S.; Krishna, M. Percentage prevalence of patient and visitor violence against staff in high-risk UK medical wards. Clin. Med. 2013, 13, 543-546. [CrossRef] [PubMed]

88. Shafran-Tikva, S.; Zelker, R.; Stern, Z.; Chinitz, D. Workplace violence in a tertiary care Israeli hospital-A systematic analysis of the types of violence, the perpetrators and hospital departments. Isr. J. Health Policy Res. 2017, 6, 43. [CrossRef]

89. Guay, S.; Goncalves, J.; Jarvis, J. A systematic review of exposure to physical violence across occupational domains according to victims' sex. Aggress. Violent Behav. 2015, 25, 133-141. [CrossRef]

90. Farrell, G.A.; Shafiei, T.; Chan, S.P. Patient and visitor assault on nurses and midwives: An exploratory study of employer 'protective' factors. Int. J. Ment. Health Nurs. 2014, 23, 88-96. [CrossRef]

91. Wang, N.; Wu, D.; Sun, C.; Li, L.; Zhou, X. Workplace Violence in County Hospitals in Eastern China: Risk Factors and Hospital Attitudes. J. Interpers. Violence 2018. [CrossRef]

92. Hasan, M.I.; Hassan, M.Z.; Bulbul, M.M.I.; Joarder, T.; Chisti, M.J. Iceberg of workplace violence in health sector of Bangladesh. BMC Res. Notes 2018, 11, 702. [CrossRef] 